\title{
VECTOR SUBSERIES OF MAXIMUM MODULUS
}

\section{HUGH J. HAMILTON}

This note extends to Euclidean $n$-space $E_{n}$ for $n \geqq 2$ and to the generalized Euclidean space $E_{\infty}$ certain investigations undertaken in [1] ${ }^{1}$ with reference to $E_{2}$. Among other things, we shall show that, given any absolutely convergent series $\sum c_{k}$ of elements in a Banach space, there is a subseries $\sum c_{j}^{*}$ of maximum modulus; that in $E_{n}$ for $n \geqq 2$ this maximum is greater than $\rho \sum\left|c_{k}\right|$, where the "best possible" value for $\rho$ is $\Gamma(n / 2) /\left\{2 \pi^{1 / 2} \Gamma[(n+1) / 2]\right\}$; and that in $E_{\infty}$ the corresponding quantity $\rho$ is zero. We shall also obtain certain related results in $E_{n}$ and in $E_{\infty}$ for series $\sum b_{k}$ with $\sum\left|b_{k}\right|=\infty$.

The value of $\rho$ in $E_{n}$ is not new, although its inaccessibility seems to have been ignored. It was obtained in 1938 by A. E. Mayer [2] for finite series, and again in 1943 by H. Hadwiger [3] as a corollary to a general theorem on "direction-functionals."

Since many of our present proofs are strictly parallel to proofs in [1], we shall make our symbols conform as closely as possible to those in that paper, shall use freely the theory of multi-dimensional analytic geometry, and shall generally omit proofs where the corresponding proofs in [1] are sufficiently suggestive. (Many of these proofs can be constructed from those in [1] by merely replacing the variable angle $\phi$ by a variable unit vector and the element of integration $d \phi$ by the element of $n$-dimensional solid angle.) We shall denote components of vectors by superscripts on the symbols for the vectors themselves, let $x$ represent a variable unit vector, use the summation convention with respect to repeated superscripts (thus $x^{i} b_{\mathbf{k}}^{i}$ signifies $\left.\sum_{i} x^{i} b_{\mathbf{k}}^{j}\right)$, use abbreviations such as $B_{k} \equiv\left|b_{k}\right|$ and $\left(x, b_{k}\right)$ $\equiv \arccos \left(x^{i} b_{k}^{i} / B_{k}\right)$, and insert a " $(+)$ " before a summand or integrand to denote summation or integration over positive values only. Unless we specifically assert that any particular discussion relates to $E_{n}$ or to $E_{\infty}$, it is to be assumed to relate to both.

Let $\mathfrak{A}$ be the class of absolutely convergent series, finite or infinite, $\sum a_{k}$ (denoted alternatively by $A$ ) the class of nonzero vectors in $E_{n}(n \geqq 2)$ or in $E_{\infty}$, and $\sum a_{j}^{\prime}$ (denoted alternatively by $S$ ) the general subseries of $\sum a_{k}$ for fixed $\sum a_{k}$. Let $B$ be the class of series $\sum b_{k}$ (denoted alternatively by $B$ ) of nonzero vectors, in the same space, for which $\sum\left|b_{k}\right|=\infty$, and $\sum b_{j}^{\prime}$ (denoted alternatively by $T$ ) the general subseries of $\sum b_{k}$ for fixed $\sum b_{k}$.

Received by the editors November $28,1949$.

${ }^{1}$ Numbers in brackets refer to the references cited at the end of the paper. 
THEOREM 1. Given an absolutely convergent series $\sum c_{k}$ of elements in a Banach space, there exists a subseries $\sum c_{j}^{*}$ of maximum modulus.

Proof. If the proposition is false, then there exists a sequence of subseries $\sum_{j} c_{j}^{\prime(\nu)}(\nu=1,2, \cdots)$ whose moduli tend to the (finite) supremum of the moduli of all subseries $\sum c_{j}^{\prime}$ of $\sum c_{k}$. Let successive subsequences of this sequence be chosen so that: in the first, $c_{1}$ is a term in each series or in none; in the second, $c_{2}$ is a term in each series or in none; and so on. Defining $\epsilon_{n}$ as 1 if $c_{n}$ appears in the series of each subsequence after the $n$th step in this process, and as 0 otherwise, we conclude that the modulus of each series in the $n$th subsequence differs from that of $\sum \epsilon_{k} c_{k}$ by not more than $\sum_{k>n}\left|c_{k}\right|$; and since this tends to zero as $n$ becomes infinite, we see that $\left|\sum \epsilon_{k} c_{k}\right|$ equals the aforementioned supremum. The conclusion follows.

Returning to $E_{n}$ and $E_{\infty}$, we define

$$
F(x) \equiv \sum(+) A_{k} \cos \left(x, a_{k}\right) \quad \text { for all } x .
$$

In what follows, up to and including Theorem $3, \sum a_{k}$ will signify an arbitrary but fixed series of class $\mathfrak{A}$.

THEOREM 2. Let $\sum \bar{a}_{j}$ be a subseries of $\sum a_{k}$ for which $\left|\sum \bar{a}_{j}\right|$ $=\max _{s}\left|\sum a_{j}^{\prime}\right|$, and let $\bar{x} \equiv \sum \bar{a}_{j} /\left|\sum \bar{a}_{j}\right|$. Then sup $F(x)=\left|\sum \bar{a}_{j}\right|$ $=F(\bar{x})$.

Proof. ${ }^{2}$ Let $x$ be arbitrary and $\sum a_{j}^{\prime}$ the sum of those terms of $\sum a_{k}$ for which $\cos \left(x, a_{k}\right)>0$. Then

$$
\begin{aligned}
F(\bar{x}) & =\sum(+) A_{k} \cos \left(\bar{x}, a_{k}\right) \geqq \sum \bar{A}_{j} \cos \left(\bar{x}, \bar{a}_{j}\right)=\left|\sum \bar{a}_{j}\right| \geqq\left|\sum a_{j}^{\prime}\right| \\
& \geqq \sum(+) A_{k} \cos \left(x, a_{k}\right)=F(x) .
\end{aligned}
$$

COROLlary 2.1. In the notation of Theorem 2, $\sum \bar{a}_{j}$ is the sum of those terms of $\sum a_{k}$ for which $\cos \left(\bar{x}, a_{k}\right)>0$.

THEOREM $^{3} 2 \mathrm{a}$. Let $x^{*}$ be such that $F\left(x^{*}\right)=\max F(x)$, and let $\sum a_{j}^{*}$ be the sum of those terms of $\sum a_{k}$ for which $\cos \left(x^{*}, a_{k}\right)>0$. Then $\max _{S}\left|\sum a_{j}^{\prime}\right|=F\left(x^{*}\right)=\left|\sum a_{j}^{*}\right|$.

${ }^{2}$ We insert this proof only to demonstrate the sense in which henceforth omitted proofs are essentially identical with the corresponding proofs in [1]. Those who are unfamiliar with the techniques of multi-dimensional analytic geometry will observe that the "geometrically obvious" inequality $\left|\sum a_{i}^{\prime}\right| \geqq \sum(+) A_{k} \cos \left(x, a_{k}\right)$ is merely the Cauchy-Schwartz inequality $\left[\left(\sum a_{j}^{\prime}\right) /\left|\sum a_{j}^{\prime}\right|\right]^{i}\left(\sum a_{j}^{\prime}\right)^{i} \geqq x^{i}\left(\sum a_{j}^{\prime}\right)^{i}$.

${ }^{3}$ This is the counterpart of Theorem 1 of [1]. Its inclusion after the present Theorem 1 is due to the fact that we cannot show by the method of [1] that $F(x)$ attains its supremum in $E_{\infty}$, since $E_{\infty}$ is not locally compact. 
COROLlaRY 2a.1. In the notation of Theorem $2 \mathrm{a}, x^{*}=\sum a_{j}^{*} /\left|\sum a_{j}^{*}\right|$.

TheOREм 3. In the notation of Theorem 2, there exists no term $a_{l}$ of $\sum a_{k}$ for which $\cos \left(\bar{x}, a_{l}\right)=0$.

We now define $\rho \equiv \inf _{A} \max _{S}\left|\sum a_{j}^{\prime}\right| / \sum A_{k}$.

TheOREM 4. In $E_{n}, \rho=\Gamma(n / 2) /\left\{2 \pi^{1 / 2} \Gamma[(n+1) / 2]\right\}$; in $E_{\infty}, \rho=0$.

Proof. In $E_{\infty}$ we let the positive integer $N$ be arbitrary and consider the series of $N$ terms, $\sum a_{k} \equiv(1,0,0, \cdots, 0,0,0, \cdots)$ $+(0,1,0, \cdots, 0,0,0, \cdots)+(0,0,1, \cdots, 0,0,0, \cdots)+\cdots$ $+(0,0,0, \cdots, 0,1,0, \cdots)$. It is clear that the subseries $\sum a_{j}^{*}$ of maximum modulus is $\sum a_{k}$ itself; and we have $\left|\sum a_{j}^{*}\right| / \sum A_{k}$ $=N^{1 / 2} / N=1 / N^{1 / 2}$.

In $E_{n}$ we integrate $F(x)$ with respect to $\omega$ over $S$, where $\omega$ is the solid angle, whose element $d \omega \equiv d x^{2} d x^{3} \cdots d x^{n} / x^{1}\left(\sum x^{i^{2}}=1\right)$ is inariant under rotation, and where $S$ is the surface of the unit sphere. Thus $\int_{S} F(x) d \omega=\sum A_{k} \int_{S}(+) \cos \left(x, a_{k}\right) d \omega$. But if for each $k$ we introduce a rotation $\xi^{j}=b_{j}^{i} x^{i}$ in which $b_{1}^{i}=a_{k}^{i} / A_{k}$, we have $\int_{S}(+) \cos$ $\left(x, a_{k}\right) d \omega=\int_{S}(+) \xi^{1} d \omega=\int_{S: \xi^{1}>0} d \xi^{2} d \xi^{3} \cdots d \xi^{n}=V_{n-1}$, where $V_{n-1}$ is the volume of the unit sphere in $(n-1)$-space. So $\int_{S} F(x) d \omega=V_{n-1} \sum A_{k}$. And since also $S=\int_{S} d \omega$, we have $\max F(x) \geqq \sum A_{k}\left(V_{n-1} / S\right)$, so that, by Theorem $2 \mathrm{a}, \rho \geqq V_{n-1} / S$, which has the value asserted for $\rho$ in the theorem [4].

We now divide $S$ into portions $\Delta_{k} S$ of maximum diameter $\delta$, choose for each $k$ the position vector $\xi_{k}$ of an arbitrary point in $\Delta_{k} S$, and consider the finite series $\sum a_{k} \equiv \sum \xi_{k} \Delta_{k} S$. Then $F(x)=\sum(+) \cos$ $\left(x, \xi_{k}\right) \Delta_{k} S$. Letting $\epsilon>0$ be arbitrary, we choose $\delta$ such that

$$
\left|\sum(+) \cos \left(x, \xi_{k}\right) \Delta_{k} S-\int_{S}(+) \cos (x, \xi) d \omega\right|<\epsilon
$$

for all $x$. [To see that this is possible we observe that for each $x$ a $\delta \equiv \delta(x)$ exists for which (1) is true and then deduce the desired uniformity by contradiction.] But under a rotation from $\xi^{i}$ coordinates to $\eta^{j}$ coordinates in which $\eta^{1}=x^{i} \xi^{i}$ the integral in (1) reduces to $V_{n-1}$, and since $\sum A_{k}=\sum\left|\xi_{k} \Delta_{k} S\right|=S$, we have $\left|F(x)-\sum A_{k}\left(V_{n-1} / S\right)\right|<\epsilon$ for all $x$. This completes the proof.

TheOREm 5. There exists no series $\sum a_{k} \in \mathfrak{A}$ for which $F(x)$ is constant.

THEOREM 6. Given an arbitrary sequence, finite or infinite, of pairs $\left(C_{k}, x_{k}\right)$, where the $x_{k}$ are unit vectors and the $C_{k}$ are positive numbers with $\sum C_{k}<\infty$, then $\Phi(x) \equiv \sum C_{k}\left|x^{i} x_{k}^{i}\right|$ is not constant. 
Proof. The series $\sum a_{k}$ defined thus:

is of class $\mathscr{A}$, and

$$
a_{2 k-1} \equiv C_{k} x_{k}, a_{2 k} \equiv-C_{k} x_{k}
$$

$$
\begin{aligned}
F(x) & =\sum(+) C_{k} \cos \left(x, x_{k}\right)+\sum(+)\left[-C_{k} \cos \left(x, x_{k}\right)\right] \\
& =\sum C_{k}\left|x^{i} x_{k}^{i}\right|=\Phi(x) .
\end{aligned}
$$

The conclusion now follows from Theorem 5 .

THEOREM 7. There exists no series $\sum a_{k} \in \mathfrak{A}$ for which it is true that $\max _{S}\left|\sum a_{j}^{\prime}\right| / \sum A_{k}=\rho$.

Proof. In $E_{\infty}$ this is obvious. In $E_{n}$ the proof proceeds as in [1].

THEOREM 8. Let $\sum b_{k} \in \mathfrak{B}$ be arbitrary. There exists a subseries $\sum b_{j}^{*}$ of $\sum b_{k}$ for which ${ }^{4}$

$$
\begin{aligned}
\underset{N}{\lim \sup }\left|\sum^{\prime} b_{j}^{*}\right| / \sum_{1}^{N} B_{k} & =\sup _{T} \lim _{N} \sup _{N}\left|\sum^{\prime} b_{j}^{\prime}\right| / \sum_{1}^{N} B_{k} \\
& =\limsup _{N} \sup _{T}\left|\sum^{\prime} b_{j}^{\prime}\right| / \sum_{1}^{N} B_{k} \\
& =\lim \sup _{N} \max _{T}\left|\sum^{\prime} b_{j}^{\prime}\right| / \sum_{1}^{N} B_{k} .
\end{aligned}
$$

We now define $\sigma \equiv \inf _{B} \max _{T} \lim \sup _{N}\left|\sum b_{j}^{\prime}\right| / \sum_{1}^{N} B_{k}$.

LEMMA 9.1. $\sigma \geqq \rho$.

Before proving Lemma 9.2 (below), we make the following constructions.

In $E_{\infty}$ we define $b_{1} \equiv(1,0,0, \cdots), b_{2} \equiv(0,1,0, \cdots), \cdots$.

In $E_{n}$, for each $\nu=1,2, \cdots$, we divide $S$ into portions $\Delta_{k}^{(\nu)} S$ of maximum diameter $\delta^{(v)}$, choose for each $k$ the position vector $\xi_{\boldsymbol{z}}^{(v)}$ of an arbitrary point in $\Delta_{k}^{(v)} S$, and let $\delta^{(v)}$ be so small that

$$
\left|\sum(+) x^{i} \xi_{k}^{(p) i} \Delta_{k}^{(\nu)} S-\int_{S}(+) x^{i} \xi^{i} d \omega\right|<S / 2^{\prime} \quad \text { for all } x .
$$

(Compare the proof of Theorem 4.) Let $N$, be the number of portions into which $S$ is thus divided and define the series $\sum b_{k}$ of class $\mathfrak{B}$ in such a way that the first $N_{1}$ terms are the $\xi_{\mathfrak{k}}^{(1)} \Delta_{\mathfrak{k}}^{(1)} S$ in any order, the next $N_{2}$ terms are the $\xi_{k}^{(2)} \Delta_{k}^{(2)} S$ in any order, and so on.

4 The notation $\Sigma$ ' indicates summation over precisely those terms of the subseries which occur among the terms of the original series summed elsewhere in the formula. 
We now define, for each $x$,

$$
F_{N}(x) \equiv \sum_{1}^{N}(+) b_{k}^{i} x^{i} / \sum_{1}^{N} \bar{B}_{k}
$$

LEMMA 9.2. $\operatorname{Lim} F_{N}(x)=\rho$ uniformly.

Proof. In $E_{\infty}$ we let $N$ be any positive integer. Then $F_{N}(x)$ $=\sum_{1}^{N}(+) x^{k} / N \leqq \sum_{1}^{N}\left|x^{k}\right| / N \leqq N^{1 / 2} / N=1 / N^{1 / 2}$.

In $E_{n}$ we let $N$ be any positive integer not less than $N_{1}$ and suppose that $N_{\nu} \leqq N<N_{\nu+1}$. Then

$$
\begin{aligned}
\left|F_{N}(x)-\rho\right| \leqq & \left|\sum_{\mu \leqq \nu}(+) x^{i} \xi_{k}^{(\mu) i} \Delta_{k}^{(\mu)} S /(\nu+\eta) S-V_{n-1} / S\right| \\
& +S /(\nu+\eta) S,
\end{aligned}
$$

where $0 \leqq \eta<1$. Hence, by (2),

$$
\begin{aligned}
\left|F_{N}(x)-\rho\right| \leqq & \left|\nu \int_{S}(+) x^{i \xi i} d \omega /(\nu+\eta) S-V_{n-1} / S\right| \\
& +S\left(1 / 2+1 / 4+\cdots+1 / 2^{\nu}\right) /(\nu+\eta) S+1 /(\nu+\eta) \\
\leqq & \left|\nu V_{n-1} /(\nu+\eta) S-V_{n-1} / S\right|+2 /(\nu+\eta),
\end{aligned}
$$

and the conclusion follows.

THEOREM 9. $\sigma=\rho$.

THEOREM 10. There exist an uncountably infinite number of subseries $\sum b_{j}^{*}$ of the series $\sum b_{k}$ of the proof of Lemma 9.2 for which

$$
\lim _{N}\left|\sum^{\prime} \bar{b}_{j}^{*}\right| / \sum_{1}^{N} \bar{B}_{k}=\max _{T} \lim _{N} \sup \left|\sum^{\prime} \bar{b}_{j}^{\prime}\right| / \sum_{1}^{N} \bar{B}_{k}=\rho=\sigma .
$$

Proof. In $E_{\infty}$ each subseries $\sum b_{j}^{\prime}$ of $\sum b_{k}$ is a subseries $\sum b_{j}^{*}$ of the type required. (Compare the first part of the proof of Theorem 4.) In $E_{n}$ the proof proceeds as in [1].

REMARKS. The formula of Theorem 4, which of course provides again the value $\rho=1 / \pi$ in $E_{2}$ which was derived in [1], also gives $\rho=1 / 2$ in $E_{1}$, which is correct, although $\inf _{A} \sup _{S}\left|\sum a_{j}^{\prime}\right| / \sum A_{k}$ is attainable in $E_{1}$. Since finally, as noted by A. E. Mayer [2], $\rho$ as a function of $n$ is asymptotically equal to $1 /(2 \pi n)^{1 / 2}$, we have an intuitive verification of the situation in $E_{\infty}$.

\section{REFERENCES}

1. H. J. Hamilton, Some theorems on subsequences, Bull. Amer. Math. Soc. vol. 44 (1938) pp. 298-304. 
2. A. E. Mayer, Grösste Polygone mit gegebenen Seitenvektoren, Comment. Math. Helv. vol. 10 (1938) pp. 288-301.

3. H. Hadwiger, Über eine Mittelwertformel für Richtungsfunktionale im Vektorraum und einige Anwendungen, J. Reine Angew. Math. vol. 185 (1943) pp. 241-252.

4. R. Courant and E. J. McShane, Differential and integral calculus, Interscience, 1947, vol. 2, pp. 303-304.

Los Angeles City College

\section{ON SETS OF CONSTANT WIDTH}

\section{GERALD FREILICH}

Sets of constant width in $E^{n}$ ( $E^{n}$ will denote Euclidean $n$-space) form an important subset of the set of all convex sets in $E^{n}$. For example, the Hausdorff $k$ measure $(0<k \leqq n)$ of a subset of $E^{n}$ is usually defined in terms of coverings of this set by arbitrary sets. However, because of the property that an arbitrary set is contained in a set of constant width of the same diameter, it is possible to refine this definition so that we need only consider coverings by sets of constant width. Unfortunately one cannot further refine the definition of Hausdorff measure, say to coverings by spheres, since it is not true that an arbitrary set is contained in a sphere of the same diameter. Thus in computing Hausdorff measures, the problem arises to find properties of sets of constant width that are analogous to those of spheres. It is the purpose of this paper to prove such a property; namely we establish a uniformity condition on sets of constant width (which incidentally does not hold for arbitrary convex sets). Roughly speaking, this uniformity condition states that parallel crosssections of a set of constant width vary continuously and that the variation for sets of given diameter $d$ can be characterized independent of the particular set. Though the motivation given above for theorems of this type arises from measure theory, I think that the results are interesting from a purely geometric point of view.

For the properties of convex sets and, in particular, sets of constant width, that will be used in this paper, the reader is referred to Theorie der konvexen Körper by J. Bonnesen and W. Fenchel, Ergebnisse der Mathematik, vol. 3, part 1, 1934. We shall in addition use the following notation:

As usual, the symbol $\rightarrow$ will denote implication between sen-

Received by the editors August 3, 1949 and, in revised form, October 25, 1949. 